\title{
Sensing Depth Analysis of Open-Ended Coaxial Probe for Skin Cancer Detection
}

\author{
Cemanur Aydinalp, Sulayman Joof, Tuba Yilmaz \\ Department of Electronics and Communication Engineering \\ Istanbul Technical University \\ Istanbul, Turkey \\ \{aydinalp16, joof, abdolsahe\}@itu.edu.tr
}

\begin{abstract}
Inherent dielectric properties discrepancy between different tissues have long been of interest to researchers in order to improve medical diagnostic approaches and therapeutic technologies. Hence, determination of the dielectric properties for biological tissues is keystone to develop an innovative medical system. Widely, the dielectric properties of biological tissues have been determined by utilizing slim form open-ended probes. Although, the method is superior to other dielectric property measurement techniques in several aspects, the measurement procedure suffers from equipment-related and tissuerelated errors. Sensing depth, which is one of the confounders can be associated with both equipment- and tissue-related errors. In this work, we performed the preparation of skin mimicking phantom and conducted a series of experiments on a two-layer configuration consisting of the prepared phantom and a liquid (olive oil). Furthermore, the sensing depth of the 2.2mm aperture open-ended coaxial probe was analyzed at five different frequencies. The results obtained show that the sensing depth of biological tissues strongly depends on the operation frequency. We also observed that the sensing depth decreases at higher frequencies and this is a property can be utilized in tackling thin multi-layered structures problem such as skin cancer detection.
\end{abstract}

Index Terms-dielectric properties, phantom, open-ended coaxial probe

\section{INTRODUCTION}

Based on the dielectric properties of biological tissues, many studies have been conducted to design diagnostic and therapeutic electromagnetic applications for different types of cancer [1]-[3]. The detection of dielectric properties contrasts which exist between malignant, benignant and normal tissues has been of interest for past decades [4], [5]. Open-ended coaxial probe method is one of the promising technique to obtain the dielectric properties from biological tissues; however, this method is developed to measure material under test that must be isotropic (uniform orientation), and homogeneous (uniform composition) [6]. Therefore, accurate detection and measurement of the target region from heterogeneous tissue is the fundamental step to retrieve the correct dielectric properties for manufacturing medical applications. While measuring the target region, the dielectric contribution of unwanted region from the heterogeneous biological tissue can be eliminated by analyzing the sensing depth measurement [7]-[9]. Although the sensing depth is not a critical issue in large-sized tumors or homogeneous tissues, sensing depth is essential for detection of target region in structures consisting of multiple layers of thin structure such as skin.

There are three main types of skin cancer: basal cell carcinoma, squamous cell carcinoma and melanoma. Despite melanoma seen much less than basal cell carcinoma and squamous cell carcinoma, melanoma aggressively metastasizes to other parts of the body over a short period of time. The major cause of melanoma is exposure to ultraviolet (UV) rays from sunlight. The DNA of genes that control skin cell growth can be damaged by UV rays, which can cause skin cancer. Lighter skinned people are at higher risk due to the lack of UV-protective melanin in their skin. For example, the incidence of melanoma is highly found in Australia, and melanoma is called 'Australia's national cancer'. Therefore, general practitioners annually consult over 1 million patients at skin cancer clinics in Australia. When deciding on a skin cancer clinic, patients consider qualifications and experience of staff, diagnosis and services offered, follow-up counseling and cost [10]. The expert utilizes dermoscopic tool and detection algorithm before transferring the patient to biopsy for histologic analysis [11]. Studies show that dielectric properties differ between carcinoma and normal healthy tissue. This discrepancy of dielectric properties can be used as a promising alternative diagnosis tool instead of digital dermoscopy and even biopsy. Present biopsy methods are costly and causes unwanted scars to patients. Therefore, the diagnostic method, which is an invasive method using dielectric will be preferred. Furthermore, more accurate information is always given to the patient during follow-up consultation by implementing this method.

In this paper, skin-mimicking phantom preparation was established and the dielectric properties of the significant steps was recorded in order to investigate how the ingredients affect dielectric properties of the phantom. Furthermore, the homogeneity of the phantom was examined at 35 different points from top to bottom. Additionally, the dielectric properties of skin phantom-olive oil double-layered configuration was measured between $500 \mathrm{MHz}-6 \mathrm{GHz}$ frequency range with slim-form open-ended coaxial probe with $2.2 \mathrm{~mm}$ diameter in an attempt to analyze the sensing depth based on five different frequencies. The rest of the paper is organized as follows, phantom preparation and 
dielectric property measurement set-up details are given in Section II, dielectric property measurement results are given in Section III, and conclusion is drawn in Section IV.

\section{Methodology}

This section details the phantom preparation steps, homogeneity analysis, and the dielectric properties measurement procedure.

\section{A. Phantom Preparation}

The quantities and the materials used for the preparation of skin phantom are listed in Table I. The ingredients of the phantom are distilled water, sunflower oil, gelatin powder, liquid detergent, $\mathrm{NaCl}$. Skin-mimicking phantom shown in Fig. 1 has been prepared by following the steps listed below [12]:

- Step 1: $40 \%$ (92g) distilled water was poured into a beaker and placed on magnetic stirrer hot plate. The water was gradually heated up, while gelatin was slowly added into the water. The mixture was stirred until the temperature reached $80^{\circ} \mathrm{C}$.

- Step 2: The gelatin-water mixture turned transparent yellow. The heater was turned off to cool the mixture until $50^{\circ} \mathrm{C}$ while continuously stirring.

- Step 3: When the mixture reached $50^{\circ} \mathrm{C}$, the remaining deionzed water $(138 \mathrm{~g})$ was added.

- Step 4: After 20 minutes, the bubbles resulting from stirring the mixture was removed with a tea strainer. $3 \mathrm{ml}$ sample was taken from the container in order to measure the dielectric properties at $36^{\circ} \mathrm{C}$.

- Step 5: $\mathrm{NaCl}$ was added in the mixture at $35^{\circ} \mathrm{C}$. After stirring for a certain time, $3 \mathrm{ml}$ sample was taken from the container in order to show how $\mathrm{NaCl}$ affects the dielectric properties.

- Step 6: While adding detergent, the speed of stirrer was slowed down in order to prevent bubbling.

- Step 7: 15 minutes later, the bubbles resulting from stirring was removed with the tea strainer. $3 \mathrm{ml}$ sample was taken from the container in order to measure the dielectric properties at $34^{\circ} \mathrm{C}$.

- Step 8: When the temperature of the mixture decreased to $28^{\circ} \mathrm{C}$, sunflower oil was added into mixture by absolutely slow gradual steps.

- Step 9: The mixture was left to stir for 45 minutes. (If ingredients are not mixed homogeneous enough, continue mixing further.) $3 \mathrm{ml}$ sample was taken from the container in order to measure the dielectric properties.

- Step 10: The mixture was poured into beakers to cool and solidify overnight at a room temperature.

The impact of phantom ingredients are shown in Fig. 2a and Fig. $2 b$ by measuring the dielectric properties of step $4,5,7,9$. The purpose of ingredients can be summarized as:

- Distilled water, sunflower oil, $\mathrm{NaCl}$ are for the modification of dielectric properties.
- Gelatin powder is utilized to control water evaporation and have semi-solid phantom structure.

- Liquid detergent (Bingo), which has permittivity of 60 and conductivity of $4.5 \mathrm{~S} / \mathrm{m}$ at $500 \mathrm{MHz}$, is used as a surfactant.

To analyze the homogeneity of the phantom, a cylindrical shaped phantom with $6.5 \mathrm{~cm}$ diameter and height was prepared and left to solidify overnight. Seven different depths from the surface to the bottom of phantom were measured, and at each depth five different measurements where taken at different positions. The permittivity and conductivity of these depths are plotted in Fig. 3. The results show that the dielectric properties of the phantom at 35 different positions appears to be consistent at $500 \mathrm{MHz}$.

TABLE I: The composition of the skin phantom.

\begin{tabular}{|c|c|}
\hline Materials & Quantity \\
\hline Distilled water & $230 \mathrm{~g}$ \\
\hline Sunflower oil & $75 \mathrm{~g}$ \\
\hline Gelatin & $34 \mathrm{~g}$ \\
\hline $\mathrm{NaCl}$ & $1.4 \mathrm{~g}$ \\
\hline Liquid detergent & $40 \mathrm{~g}$ \\
\hline
\end{tabular}

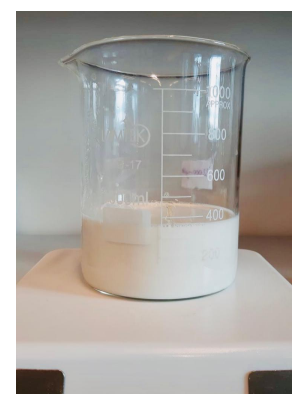

Fig. 1: Skin phantom sample.

After evaluation of the phantom at the other frequencies, we observed that the prepared phantom is homogeneous enough to utilize as a skin-mimicking tissue.

\section{B. Dielectric Properties Measurement}

The dielectric property measurement system is composed of Agilent FieldFox N9923A 6GHz RF Vector Network Analyzer, Agilent N1501A Dielectric Slim Form Probe with $2.2 \mathrm{~mm}$ aperture, Agilent $85070 \mathrm{E}$ software, an external computer, adjustable stand and a digital caliper shown in Fig 4. Agilent FieldFox N9923A 6GHz RF Vector Network is used to obtain reflection coefficients (S11-parameters) from $500 \mathrm{MHz}$ to $6 \mathrm{GHz}$ with $55 \mathrm{MHz}$ increments. The dielectric properties are computed from measured S-parameters via Agilent $85070 \mathrm{E}$ software connected on an external computer.

The double-layered configuration, which is phantomolive oil, was prepared to perform the sensing depth analysis experiments. $15 \mathrm{ml}$ liquid phantom was solidified in a beaker overnight as the first layer. To measure the thickness 


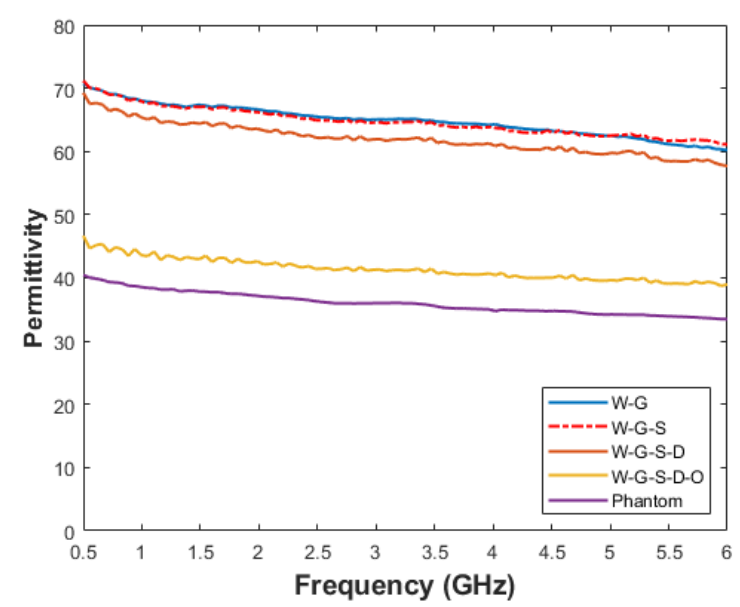

(a)

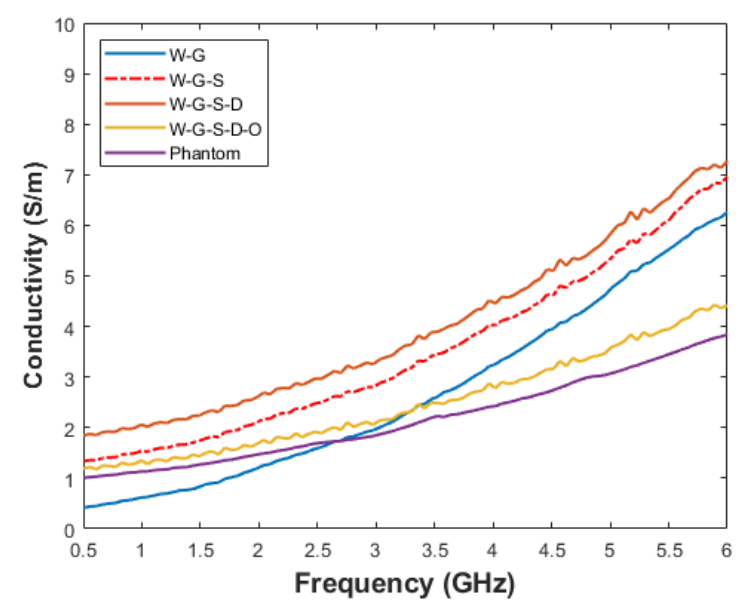

(b)

Fig. 2: (a) Permittivity of mixture for different steps and (b) conductivity of mixture for different steps.

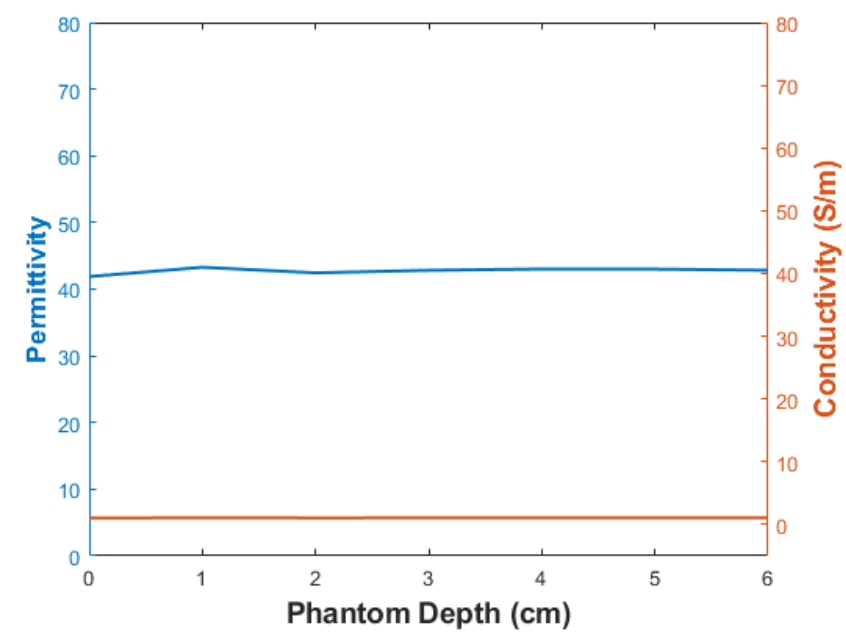

Fig. 3: Dielectric properties of $6.5 \mathrm{~cm}$ thick phantom measured at seven different depths by increment of $1 \mathrm{~cm}$ from surface to inside at $500 \mathrm{MHz}$.

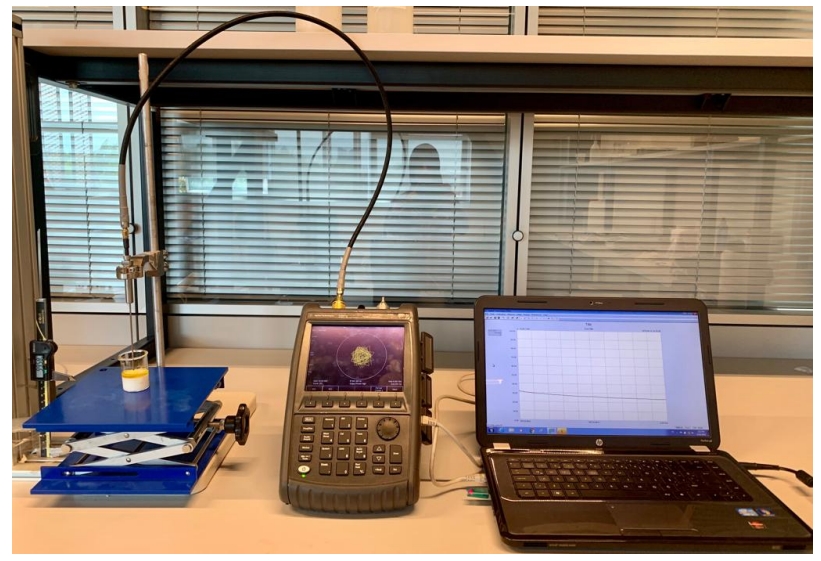

Fig. 4: Measurement set-up consists of $6 \mathrm{GHz} \mathrm{RF}$ vector network analyzer, slim form probe with $2.2 \mathrm{~mm}$ aperture, software, an external computer, adjustable stand, a digital caliper and phantom-olive oil double-layered configuration

of first layer, a digital caliper was attached to an adjustable stand to record the range of stand's movement. Using tip of the probe as a reference point, the stand was adjusted to where open-ended coaxial probe starts measuring dielectric properties of the first layer and the distance recorded on the caliper is used to calculate the thickness of the layer. The same procedure was implemented after adding olive oil as the second layer. Retrieving exact thickness of first layer and second layer, the probe was penetrated into the second layer gradually towards first layer. At each step of increment as the probe descents to the phantom, the dielectric properties measurement was obtained. When the alteration of the dielectric properties were observed, the increments of each step was minimized in order to analyze sensing depth more accurately.

\section{RESUlts}

The dielectric properties of double-layered configuration was measured using the procedure described in the previous section to analyze the sensing depth of the $2.2 \mathrm{~mm}$ open-ended coaxial probe. The dielectric properties of the phantom-olive oil double-layered configuration was measured at 101 different frequency points from $500 \mathrm{MHz}$ to $6 \mathrm{GHz}$. The obtained relative permittivity and conductivity for phantom-olive oil double-layered configuration was plotted as a function of probe distance from phantom layer for five selected frequencies: $500 \mathrm{MHz}, 1.05 \mathrm{GHz}, 2.04 \mathrm{GHz}$, $3.03 \mathrm{GHz}, 6 \mathrm{GHz}$ shown in Fig. $5 \mathrm{a}$ and $5 \mathrm{~b}$. As seen in Fig. $5 \mathrm{a}$, for instance at a distance of $220 \mu \mathrm{m}$ the dielectric properties contribution of the phantom layer to the result of dielectric property of the double-layered structure is higher at $500 \mathrm{MHz}$ compared to other frequencies. Sensing depth decreases as the frequency increases, and phantom layer contribution decreases when the probe distance is at the same distance. 
TABLE II: Dielectric properties of materials used for doublelayered configuration at $500 \mathrm{MHz}$.

\begin{tabular}{|c|c|c|}
\hline Materials & Permittivity $(\varepsilon)$ & Conductivity S/m $(\sigma)$ \\
\hline Skin Phantom & 38.26 & 0.98036 \\
\hline Olive oil & 2.56 & 0.00659 \\
\hline
\end{tabular}

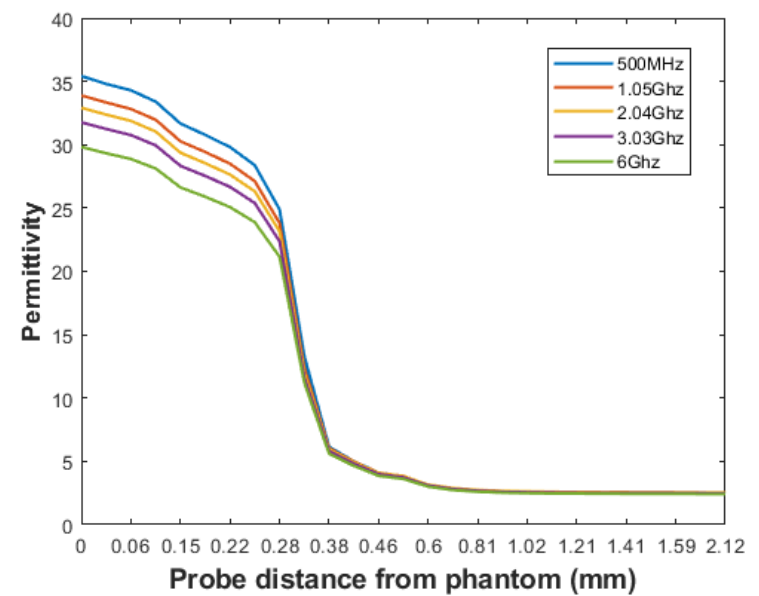

(a)

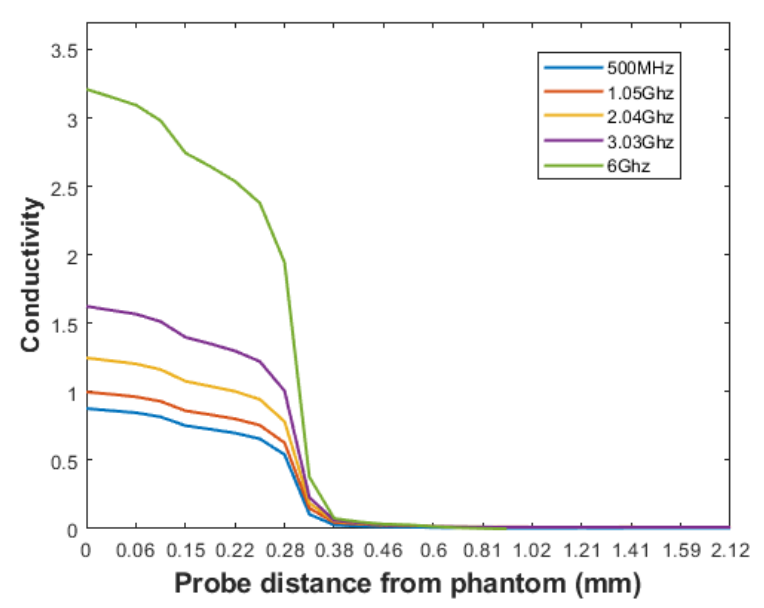

(b)

Fig. 5: (a) Permittivity of double-layered configuration and (b) conductivity of double-layered configuration for different probe distance.

Therefore, if the tissue to be diagnosed is not near to the vicinity of the probe, low frequency ranges gives a better measurement results due to the sensing depth provided by frequency range. Otherwise, if the tissue is near to the vicinity of the probe and in order to reduce the contribution of the other parts, high frequency ranges are preferable. The permittivity and conductivity of both olive oil and skinmimicking phantom used for doubled-layer configuration are listed in Table II.

\section{CONCLUSION}

The classification of biological tissues by measuring the dielectric properties will make a major contribution to the field of cancer diagnosis. Specifically, in the diagnosis of skin cancer, either the dermoscope method based on the expert's experience or the biopsy method which is inconvenient to the patient in many respects is used.Therefore, the open-ended coaxial probe method will be the preferred method in terms of both accuracy and cost-effectiveness. It is very difficult to identify the tumorous structure that occurs in biological tissue, which is thin but contains many layers, such as skin. For this reason, the sensing depth of the probe used is important. The sensing depth varies depending on the frequency used, and taking measurements in the correct frequency range, which can be easily adjusted by the user, would greatly contribute to the measurement accuracy. Instead of having a wide frequency range, it would be more reliable to design medical applications using a narrow-range frequency band where consistency is established and the discrepancy is explicit. Therefore, in this study, measurement is performed at 101 different frequency points. 5 representative frequency values were selected and compared in terms of the distance from the phantom layer.

\section{ACKNOWLEDGMENT}

This project has received funding from the European Union's Horizon 2020 research and innovation program under the Marie Sklodowska-Curie grant agreement No. 750346 and COST Action CA17115.

\section{REFERENCES}

[1] M. Lazebnik, D. Popovic, L. McCartney, C. B. Watkins, M. J. Lindstrom, J. Harter, S. Sewall, T. Ogilvie, A. Magliocco, T. M. Breslin et al., "A large-scale study of the ultrawideband microwave dielectric properties of normal, benign and malignant breast tissues obtained from cancer surgeries," Physics in Medicine \& Biology, vol. 52, no. 20, p. 6093, 2007.

[2] A. Mirbeik-Sabzevari and N. Tavassolian, "Tumor detection using millimeter-wave technology: Differentiating between benign lesions and cancer tissues," IEEE Microwave Magazine, vol. 20, no. 8, pp. 30-43, 2019.

[3] T. Yilmaz, M. A. Kılıç, M. Erdoğan, M. Çayören, D. Tunaoğlu, İ. Kurtoğlu, Y. Yaslan, H. Çayören, A. E. Arıkan, S. Teksöz et al., "Machine learning aided diagnosis of hepatic malignancies through in vivo dielectric measurements with microwaves," Physics in Medicine \& Biology, vol. 61, no. 13, p. 5089, 2016.

[4] E. C. Burdette, F. L. Cain, and J. Seals, "In vivo probe measurement technique for determining dielectric properties at vhf through microwave frequencies," IEEE transactions on microwave theory and techniques, vol. 28, no. 4, pp. 414-427, 1980.

[5] C. Gabriel, S. Gabriel, and y. E. Corthout, "The dielectric properties of biological tissues: I. literature survey," Physics in medicine \& biology, vol. 41, no. 11, p. 2231, 1996.

[6] K. N1501A. (2019) Dielectric probe kit $10 \mathrm{mhz}$ to $50 \mathrm{ghz}$. [Online]. Available: https://literature.cdn.keysight.com/litweb/pdf/59920264EN.pdf

[7] D. M. Hagl, D. Popovic, S. C. Hagness, J. H. Booske, and M. Okoniewski, "Sensing volume of open-ended coaxial probes for dielectric characterization of breast tissue at microwave frequencies," IEEE Transactions on Microwave Theory and Techniques, vol. 51, no. 4, pp. 1194-1206, 2003. 
[8] P. M. Meaney, A. P. Gregory, J. Seppälä, and T. Lahtinen, "Open-ended coaxial dielectric probe effective penetration depth determination," IEEE transactions on microwave theory and techniques, vol. 64, no. 3 , pp. 915-923, 2016.

[9] E. Porter, A. La Gioia, A. Santorelli, and M. O'Halloran, “Modeling of the dielectric properties of biological tissues within the histology region," IEEE Transactions on Dielectrics and Electrical Insulation, vol. 24, no. 5, pp. 3290-3301, 2017.

[10] C. Council. (2019) Skin cancer. [Online]. Available: https://www.cancer.org.au/about-cancer/types-of-cancer/skincancer.html

[11] G. A. Holmes, J. M. Vassantachart, B. A. Limone, M. Zumwalt, J. Hirokane, and S. E. Jacob, "Using dermoscopy to identify melanoma and improve diagnostic discrimination," Federal Practitioner, vol. 35, no. Suppl 4, p. S39, 2018.

[12] T. Yilmaz, "Towards optimization of open ended contact probes for breast cancer diagnosis," in 2018 Progress in Electromagnetics Research Symposium (PIERS-Toyama). IEEE, 2018, pp. 993-999. 